\section{PHYSICS AND CHEMISTRY OF SWELLING AND SHRINKING}

$\mathrm{T}$ HE gerreral dischs of the Faraday Society are usually notagle for the breadth of their scope, and the latest. Weld during September 24-26

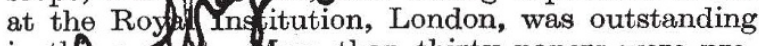
in thas afet. More than thirty papers were presentech ander the general title of "Swelling and Shrinkifg", dealing with topics ranging from the thermodynamics of liquid mixtures to the behaviour of complex biological and technical systems. These were grouped in three sections: fundamental, biological, and technical. Prof. J. D. Bernal gave a general introductory talk, and the second and third sections were introduced respectively by Prof. E. K. Rideal and by Dr. L. G. Gabriel.

In attempting to summarize briefly some of the salient features of the discussion, attention may first be directed to two diametrically opposed methods of attack. The first is concerned with the simplest possible types of system, comprising essentially nonpolar polymers and liquids, and as free as possible from structural features. At the other extreme lie certain aqueous systems which have been found by $\mathrm{X}$-ray examination to possess very definite structural regularities.

Various methods of investigation have been applied to non-polar systems, and a great deal of progress has been made in recent years in the interpretation of the thermodynamic properties of polymer liquid mixtures. Lattice models have been made the basis of statistical calculations of the heat and entropy of solution of polymers. Discussion of recent progress in this field revealed the lack of an adequate treatment of dilute solutions, taking accurate account of the extent to which a single polymer molecule is folded or crumpled by its random thermal motion. Measurements of the intensity of light scattered by a polymer solution, and its distribution about the direction of incidence, are now being used to give direct estimates of the root mean square length of a polymer. Preliminary results reported at the discussion give values some four times larger than those computed for an ideally flexible and volumeless chain, and these lengths do not appear to be very dependent on the nature of the liquid. Mixtures of higher polymer content have been treated with greater success, but evidence was presented that liquids which are only sparingly absorbed are present in the nearly saturated polymer in the form of small clusters. This behaviour, which probably has a counterpart in liquid mixtures, has not been satisfactorily explained.

Less progress has been made in the quantitative interpretation of viscous flow and diffusion in these systems, although the qualitative features observed are readily understood. An outstanding problem in calculating the viscosity of a dilute solution is to decide whether liquid is effectively trapped by the crumpled polymer chain, or flows freely through it. Both assumptions have been made, and the experimental evidence suggests an intermediate state of affairs, possibly somewhat dependent on the solvent. New evidence presented at the discussion was concerned with model suspensions of flexible particles cut from cellulose fibres and swollen. These were, of course, of much more than molecular dimensions, and liquid was observed to flow freely through their folds. Nevertheless, the viscosity was found to depend on the overall length, rather than the mean length taken up by the particles when suspended in liquid.

Several examples were discussed of systems which possess regular structures when highly swollen by water or aqueous media. One of the most remarkable of these is furnished by dilute solutions of tobacco mosaic virus. X-ray examination shows the rod-like molecules to be arranged in a regular hexagonal pattern, with a spacing which may be as much as 500 A., depending on the medium, but not on the length of the rods. Regular structures with even larger separations are observed in sols of ferric and tungstic oxides, the plate-like particles of which form 'Schiller layers' with separation as great as $8000 \mathrm{~A}$. Two types of explanation of these structures assume on one hand a supporting framework, and on the other, the existence of long-range forces. It is difficult to see how the very regular framework needed could arise, and it was generally assumed by contributors to this discussion that long-range forces must be responsible. The origin and nature of these forces is by no means clear, and much difference of opinion was expressed. The two main suggestions were that the necessary free-energy minimum arises from a balance between attractive and repulsive forces, both electrostatic in origin, or between van der Waals' attraction and electrostatic repulsion. On either view it appears difficult to account for the existence of a free-energy minimum sufficiently deep to confer reasonable stability on the structure.

Another very striking example of an aqueous system possessing structure is furnished by protein crystals. X-ray examination of horse methæmoglobin shows the swollen erystals to consist of alternate layers of hæmoglobin molecules and liquid. Swelling and shrinking produce discontinuous changes in the layer spacing and in the $\beta$ angle of the crystal. In this case the spacing does not exceed $15 \mathrm{~A}$., so that a definite structure may well extend through the complete liquid layer.

Hydrated clays, such as montmorillonite, show similar laminated structures, in which swelling and shrinking occur by variations in the thickness of the water layers. Experiments on the swelling of dehydrated montmorillonite in polar organic liquids were also reported ; as many as three molecular layers of nitromethane or acetonitrile could be intercalated between successive clay layers, the spacing then being of the order of $20 \mathrm{~A}$. Evidence was presented that these layers were essentially liquid.

A number of systems showing structure on a microscopic scale were described by various contributors to the discussion. When lecithin swells in water, the particle is observed to develop cylindrical excrescences which retain a constant diameter, and do not coalesce on contact. These have been termed 'myelin forms', and similar arrangements have been found with other systems, including the complex formed by association of cholesterol (insoluble) with lysolecithin (soluble). By using complexes of increasing solubility, it has been found possible to produce in succession a series of structural types which had been previously reported. These include (1) 'batonnets', consisting of axially symmetrical particles with characteristic bulges which coalesce on contact, giving new particles of the same type; (2) long spindleshaped 'tactoids'; (3) 'coacervates' containing spherical particles. It was suggested that the basic molecular organisation in these structures is again laminar, with the fluid content of the swollen phase intercalated between crystalline layers. 
An interesting series of transparent 50 per cent oil-water systems was described. If amyl alcohol is added to an emulsion stabilized by sodium oleate, the system becomes transparent, and is believed to contain suspended droplets of the order of 100-200 A. in diameter. Amyl alcohol can be replaced by other amphipathic compounds, their efficiency depending on the ratio polar/non-polar. This was investigated. in the series of aliphatic alcohols, from $\mathrm{C}_{1}$ to $\mathrm{C}_{10}$, and it was found that with benzene as the 'oil', the continuous phase changed from water to oil when the chain-length exceeded 5. These results are explicable in terms of the formation of mixed films of soap and alcohol at the interface ; such films are known to be stable from experiments in monolayers.

Between these two extremes of structureless and highly ordered systems lie many of considerable importance, which may be treated as approximating more or less closely to one extreme or the other. Two papers discussed the structure and deformation of cellulose gels in the light of the work on ideally elastic rubber-like bodies. Theoretical treatments of the latter have been based on a model consisting of long randomly linked chains joined at a few points into a complete three-dimensional network. A statistical mechanical analysis of this model leads to expressions for the free energy of deformation, and the orientation birefringence. Swollen cellulose shows a limited degree of rubber-like elasticity, but in order to account for this quantitatively it has been necessary to assume a very high degree of crosslinking. It may be considered doubtful whether much significance is attached to the model when the estimated number of statistical chain elements between junction points falls so low as one or two.

The swelling of nitrocellulose is more complicated than that of a purely amorphous polymer, on account of its definite structure. Two types of swelling were reported, depending on whether the crystalline part is affected or not.

The swelling of protein fibres in organic solvents was discussed from the point of view of the fibre structure. The relative effectiveness of a range of acids, amides and alcohols suggests that swelling involves the breakdown of the fibre structure by chemical interactions between the liquid and localized groups in the fibre. No simple generalization of these results, such as may be made for the swelling of nonpolar polymers, was found to be valid in this case.

The phenomena of swelling and shrinking are of great importance in many widely divergent fields. In the technical use of materials capable of absorbing water, or other liquids, the resultant change of volume may be less serious than the stresses and strains which are produced. A thermodynamic analysis of the swelling of wood was presented, in which the cell structure was represented by a hollow cylinder of initially isotropic material surrounded by a rigid sheath, so that swelling could only occur into the central lumen. It is then possible to deduce the dependence of the vapour pressure at fixed moisture content on the elastic constants of the swollen cell wall. The swelling of laminated plastics represents a closely related problem. Imbibition of moisture by the reinforcing fibre is mechanically restrained by the surrounding film of polymer, which in general will itself be swollen to a smaller degree.

A very different field of application of the general ideas developed in this conference is presented by systems of biological interest. On the simplest view, a red cell may be treated as a balloon-like body sur- rounded by a semi-permeable membrane of very low modulus of elasticity. The volume of the cell, which can now be measured with some accuracy, should thus be dependent on an osmotic equilibrium, and would vary with the medium in which the cell is immersed. Experimentally, there are found to be discrepancies which are not at present fully understood. Complications were pointed out also in the swelling of protoplasm. It is not yet clear how far the various physical processes occurring in living matter may be treated as though they are identical with those of the dead chemical constituents of the systems. Without in the least suggesting any vitalistic hypothesis, it is necessary to bear in mind the essentially dynamic nature of living cells, and the simultaneous occurrence of complex chemical and physical changes.

Geoffrey GeE

\section{THE MOON AND PLANT GROWTH}

\section{- By Dr. C. F. C. BEESON, C.I.E. Imperial Foltestry Bureau, Oxford}

BELIEFS the phases of the moon have a plants are both ancient and world-wide. Proof by rationgl efperiment seems to have been sought more - than wo hundred and fifty years ago by La Quintion the horticulturist, and some years later by Duthamel du Monceau ${ }^{1}$, the forester. Neither obtained any positive evidence of lunar influence. Since then, scientific interest in the subject has been revived intermittently, either by the 'rediscovery' of lunar rites in the agriculture of civilized countries, or by the impact on Europeans of the impressive faith of primitive peoples, particularly in the tropies and sub-tropics.

The literature on the moon and plants can be assigned to two groups : one comprising reiterations of peasant beliefs, myths and rules, both ancient and modern, and similar unsubstantiated statements ; the other comprising experiments supported by numerical data capable of statistical analysis. This second group consists of $(a)$ experiments mainly of the anthroposophical school, which demonstrate the existence of lunar effects on the growth of plants ; and $(b)$ experiments of professional horticulturists and foresters, which prove that there are no such effects, or that, if they do. exist, they have no value in agricultural practice.

The beliefs which dominate primitive rural economy and the emphatic reports of credulous observers are very numerous, but they provide no significant evidence. Only experimental data need be considered; they may be briefly summarized as follows :

(a) Kolisko's work. According to the investigations of L. Kolisko ${ }^{2}$ in Stuttgart during 1926-35, the particular phase of the moon at the time of sowing does influence the period and the percentage of germination, as also the subsequent growth of the plant. The most favourable date to sow is two days before the full moon for leaf-and fruit-bearing garden crops (such as cabbages, peas, tomatoes), for rootcrops (such as radishes, beetroots, carrots), for flowering garden annuals, and for wheat, maize, etc. In general, these plants show better germination, more vigorous growth, and greater yields than those sown just before the new moon. Kolisko affirms that the lunar influence is not fully effective unless there is rain or artificial watering during the germination period, but the stimulus once acquired remains 